\title{
STOCHASTIC INTEGRAL AND SERIES REPRESENTATIONS FOR STRICTLY STABLE DISTRIBUTIONS
}

\author{
MAKOTO MAEJIMA ${ }^{1,2}$, JAN ROSIŃSKI $^{3,4}$, AND YOHEI UEDA ${ }^{5,6}$
}

\begin{abstract}
In this paper we find and develop a stochastic integral representation for the class of strictly stable distributions. We establish an explicit relationship between stochastic integral and shot-noise series representations of strictly stable distributions, which shows that the class of distributions representable by stochastic integral is larger than the class representable by a shot-noise series. This inclusion is proper when the stability index $\alpha$ is greater than 1 . We also give an explicit description of distributions possessing both representations.
\end{abstract}

\section{InTRODUCTION}

Throughout the paper, $I\left(\mathbb{R}^{d}\right)$ denotes the class of all infinitely divisible distributions on $\mathbb{R}^{d}, \mathcal{L}(X)$ stands for the distribution of a random variable $X$, and the point-mass distribution at $a \in \mathbb{R}^{d}$ is denoted by $\delta_{a}$. Given $\mu \in I\left(\mathbb{R}^{d}\right),\left\{X_{t}^{(\mu)}\right\}$ will denote a Lévy process such that $\mathcal{L}\left(X_{1}\right)=\mu$. For a fixed nonrandom function $f$, consider the stochastic integral mapping $\Phi_{f}: \mathfrak{D}\left(\Phi_{f}\right) \rightarrow I\left(\mathbb{R}^{d}\right)$ given by

$$
\Phi_{f}(\mu)=\mathcal{L}\left(\int_{0}^{\infty} f(t) d X_{t}^{(\mu)}\right)
$$

where the domain $\mathfrak{D}\left(\Phi_{f}\right)$ consists of all $\mu \in I\left(\mathbb{R}^{d}\right)$ for which the stochastic integral in (1.1) is definable (see [20]).

Stochastic integral mappings give probabilistic representations for many useful classes of distributions contained in $I\left(\mathbb{R}^{d}\right)$. Namely, such classes can be represented

Date: August 10, 2021.

2000 Mathematics Subject Classification. 60E07.

Key words and phrases. strictly stable distribution, stochastic integral representation, series representation.

${ }^{1}$ Department of Mathematics, Keio University, 3-14-1, Hiyoshi, Kohoku-ku, Yokohama 223-8522, Japan. maejima@math.keio.ac.jp

${ }^{2}$ Maejima's research was partially supported by JSPS Grant-in-Aid for Science Research 22340021.

${ }^{3}$ Department of Mathematics, 227 Ayres Hall, University of Tennessee, Knoxville, TN, USA. rosinski@math.utk.edu

${ }^{4}$ Rosiński's research was partially supported by the Simons Foundation grant 281440.

${ }^{5}$ Department of Mathematics, Keio University, 3-14-1, Hiyoshi, Kohoku-ku, Yokohama 223-8522, Japan. ueda@math.keio.ac.jp

${ }^{6}$ Corresponding author. 
as ranges of the mappings $\Phi_{f}$ for some specific functions $f$. Examples of classes of distributions having stochastic integral representations include the class of selfdecomposable distributions [9, 25, 26], the Jurek class [8], the Goldie-Steutel-Bondesson class and the Thorin class [5], the class of type $G$ distributions [2], and many other classes [1, 3, 11, 21]. Characterizations of the domains and ranges of the corresponding stochastic integral mappings allow to determine the extent of such representations. They are also necessary for the study of iterations of stochastic integral mappings and their limits [10, 11, 12, 13, 14, 21, 22, 23, 24], compositions of several mappings [5, 19, 12, 21], and related considerations [4, 7].

Surprisingly for the present authors, the form of a stochastic integral representation of the class of stable distributions has been unknown. On the other hand, a shot noise-type series representation of such distributions has been known and used by many authors for a long time (see, e.g., [17]). In this paper, we give a stochastic integral representation of strictly stable distributions on $\mathbb{R}^{d}$, characterize the domain and range of the corresponding stochastic integral mapping, and establish an explicit relationship between stochastic integral and series representations. Our main results are Theorems 3.3, 3.4, and 3.7.

The stochastic integral representation of strictly stable distributions is given by the mapping $\Xi_{\alpha}(\mu):=\mathcal{L}\left(\int_{0}^{\infty} t^{-1 / \alpha} d X_{t}^{(\mu)}\right)$. In Theorem 3.3 we show that the range of $\Xi_{\alpha}$ coincides with the class of strictly $\alpha$-stable distributions when $\alpha \neq 1$. The case $\alpha=1$ is more delicate since the range of $\Xi_{1}$ is smaller than the class of strictly 1-stable distributions. We describe precisely distributions representable by $\Xi_{1}$, from which it follows that every strictly 1-stable distribution belongs to the range of $\Xi_{1}$ after certain deterministic translation.

The relationship between stochastic integral and series representations of strictly stable distributions is captured when one studies the restriction of $\Xi_{\alpha}$ to the subdomain consisting of compound Poisson distributions. For such distributions $\mu$, $X_{t}^{(\mu)}=\sum_{j: \tau_{j} \leq t} V_{j}$, where $\tau_{j}$ is the $j$ th arrival time of a Poisson process and $\left\{V_{j}\right\}$ is a sequence of i.i.d. random variables in $\mathbb{R}^{d}$, independent of $\left\{\tau_{j}\right\}$. In this case, at least formally, we can write

$$
\int_{0}^{\infty} t^{-1 / \alpha} d X_{t}^{(\mu)}=\sum_{j=1}^{\infty} \tau_{j}^{-1 / \alpha} V_{j} .
$$

The right-hand side is a well-known series representation of stable distributions; see, e.g., Corollary 4.10 of Rosinski [15], Proposition 1.4.1 of [17]. In Theorem 3.4 we 
investigate 1.2 and characterize the class of distributions representable by either side of this equation. It follows that the series representation (without centering) is a special case of our stochastic integral representation.

It is clear that $\Xi_{\alpha}$ is not injective on its whole domain. In Theorem 3.7 we consider $\Xi_{\alpha}$ restricted to subdomains of some infinitely divisible distributions with finite Lévy measures supported by the unit sphere. On such subdomains, $\Xi_{\alpha}$ is injective and we characterize the corresponding ranges of $\Xi_{\alpha}$.

One special property of $\Xi_{\alpha}$ is mentioned at the end of the paper in Remark 5.2. It says that the limit of the ranges of iterations of our mapping consists of only one distribution $\delta_{0}$. This property makes $\Xi_{\alpha}$ quite different from other stochastic integral mappings considered in the past.

Finally, we remark that, as far as representations are concerned, the restriction to strictly stable distributions is not essential when $\alpha \neq 1$, because any $\alpha$-stable distribution is strictly stable up to a convolution with a $\delta$-distribution. (Theorem 14.7 in Sato [18]. See also the end of the section 2.)

\section{Preliminaries}

The characteristic function $\widehat{\mu}(z), z \in \mathbb{R}^{d}$, of $\mu \in I\left(\mathbb{R}^{d}\right)$ is given by the LévyKhintchine triplet $(A, \nu, \gamma)$ as follows

$$
\widehat{\mu}(z)=\exp \left\{-\frac{1}{2}\langle z, A z\rangle+\int_{\mathbb{R}^{d}}\left(e^{i\langle z, x\rangle}-1-i\langle z, x\rangle \mathbb{1}_{\{|x| \leq 1\}}(x)\right) \nu(d x)+i\langle\gamma, z\rangle\right\},
$$

where $A$ is a $d \times d$ symmetric nonnegative-definite matrix, $\nu$ is a measure (called the Lévy measure of $\mu$ ) on $\mathbb{R}^{d}$ satisfying $\nu(\{0\})=0$ and $\int_{\mathbb{R}^{d}}\left(|x|^{2} \wedge 1\right) \nu(d x)<\infty$ and $\gamma \in \mathbb{R}^{d}$. We will write $\mu=\mu_{(A, \nu, \gamma)}$ to denote an infinitely divisible distribution with the Lévy-Khintchine triplet $(A, \nu, \gamma)$. If $\mu=\mu_{(A, \nu, \gamma)}$ satisfies $\int_{|x|>1}|x| \nu(d x)<\infty$, then there exists the mean $\gamma^{1} \in \mathbb{R}^{d}$ of $\mu$ such that

$$
\widehat{\mu}(z)=\exp \left\{-\frac{1}{2}\langle z, A z\rangle+\int_{\mathbb{R}^{d}}\left(e^{i\langle z, x\rangle}-1-i\langle z, x\rangle\right) \nu(d x)+i\left\langle\gamma^{1}, z\right\rangle\right\}
$$

and

$$
\gamma^{1}=\gamma+\int_{|x|>1} x \nu(d x)
$$

In this case, we will write $\mu=\mu_{\left(A, \nu, \gamma^{1}\right)_{1}}$. If $\mu=\mu_{(A, \nu, \gamma)}$ satisfies $\int_{|x| \leq 1}|x| \nu(d x)<\infty$, then there exists $\gamma^{0} \in \mathbb{R}^{d}$ (called the drift of $\mu$ ) such that

$$
\widehat{\mu}(z)=\exp \left\{-\frac{1}{2}\langle z, A z\rangle+\int_{\mathbb{R}^{d}}\left(e^{i\langle z, x\rangle}-1\right) \nu(d x)+i\left\langle\gamma^{0}, z\right\rangle\right\}
$$


and

$$
\gamma^{0}=\gamma-\int_{|x| \leq 1} x \nu(d x)
$$

We will write $\mu=\mu_{\left(A, \nu, \gamma^{0}\right)_{0}}$ in this case.

Corollary 2.1. Let $\mu=\mu_{(A, \nu, \gamma)} \in I\left(\mathbb{R}^{d}\right)$. Then $\mu_{(A, \nu, 0)_{0}}=\mu_{(A, \nu, 0)_{1}}$ if and only if $\int_{\mathbb{R}^{d}}|x| \nu(d x)<\infty, \int_{\mathbb{R}^{d}} x \nu(d x)=0$ and $\gamma=\int_{|x| \leq 1} x \nu(d x)$.

We also use the following polar decomposition of a Lévy measure $\nu$. If $\nu \neq 0$, then there exist a measure $\lambda$ on $S=\left\{x \in \mathbb{R}^{d}:|x|=1\right\}$ with $0<\lambda(S)<\infty$ and a family $\left\{\nu_{\xi}, \xi \in S\right\}$ of measures on $(0, \infty)$ such that $\nu_{\xi}(B)$ is measurable in $\xi$ for each $B \in \mathcal{B}((0, \infty)), 0<\nu_{\xi}((0, \infty)) \leq \infty$ for each $\xi \in S$ and

$$
\nu(B)=\int_{S} \lambda(d \xi) \int_{0}^{\infty} \mathbb{1}_{B}(r \xi) \nu_{\xi}(d r), \quad B \in \mathcal{B}\left(\mathbb{R}^{d} \backslash\{0\}\right) .
$$

Measure $\lambda$ is called the spherical component of $\nu$ and $\nu_{\xi}$ its radial component. If $\nu\left(\mathbb{R}^{d}\right)<\infty$, then we may and do assume that $\nu_{\xi}$ are probability measures. Indeed, consider a random vector $x \mapsto\left(\frac{x}{|x|},|x|\right)$ under probability measure $\nu / \nu\left(\mathbb{R}^{d}\right)$ and let $\nu_{\xi}$ be the conditional distribution of $|x|$ given that $\frac{x}{|x|}=\xi$. Then $\left(\lambda, \nu_{\xi}\right)$ satisfy (2.1), with $\lambda(B)=\nu\left(\left\{x \neq 0: \frac{x}{|x|} \in B\right\}\right), B \in \mathcal{B}(S)$.

Let $S_{\alpha}\left(\mathbb{R}^{d}\right)$ be the class of $\alpha$-stable distributions on $\mathbb{R}^{d}, 0<\alpha<2$. The characteristic function of $\mu \in S_{\alpha}\left(\mathbb{R}^{d}\right)$ is of the form: when $\alpha \neq 1$,

$$
\widehat{\mu}(z)=\exp \left[-\int_{S}|\langle z, \xi\rangle|^{\alpha}\left(1-i \tan \frac{\pi \alpha}{2} \operatorname{sgn}\langle z, \xi\rangle\right) \lambda_{1}(d \xi)+i\langle z, \tau\rangle\right],
$$

and when $\alpha=1$,

$$
\widehat{\mu}(z)=\exp \left[-\int_{S}\left(|\langle z, \xi\rangle|+i \frac{2}{\pi}\langle z, \xi\rangle \log |\langle z, \xi\rangle|\right) \lambda_{1}(d \xi)+i\langle z, \tau\rangle\right],
$$

where $\lambda_{1}$ is a finite measure on $S$, called the spectral measure of $\mu$, and $\tau \in \mathbb{R}^{d}$ is a shift parameter. Recall that $\mu \in S_{\alpha}\left(\mathbb{R}^{d}\right)$ is strictly stable when $\widehat{\mu}(b z)=\widehat{\mu}(z)^{b^{\alpha}}$ for all $b>0$ and $z \in \mathbb{R}^{d}$. Let $S_{\alpha}^{0}\left(\mathbb{R}^{d}\right)$ denote the class of strictly $\alpha$-stable distributions on $\mathbb{R}^{d}$. Then $\mu \in S_{\alpha}^{0}\left(\mathbb{R}^{d}\right)$ if and only if

$$
\begin{cases}\mu \in S_{\alpha}\left(\mathbb{R}^{d}\right) \text { and } \tau=0, & \text { when } \alpha \neq 1, \\ \mu \in S_{1}\left(\mathbb{R}^{d}\right) \text { and } \int_{S} \xi \lambda_{1}(d \xi)=0, & \text { when } \alpha=1 .\end{cases}
$$

(See, e.g., Theorem 14.10 in Sato [18].) 


\section{The Results}

Recall that $\left\{X_{t}^{(\mu)}\right\}$ denotes a Lévy process such that $\mathcal{L}\left(X_{1}\right)=\mu \in I\left(\mathbb{R}^{d}\right)$. Let $0<\alpha<2$. Consider an improper stochastic integral defined by

$$
\int_{0}^{\infty} t^{-1 / \alpha} d X_{t}^{(\mu)}=\lim _{\varepsilon \downarrow 0, T \uparrow \infty} \int_{\varepsilon}^{T} t^{-1 / \alpha} d X_{t}^{(\mu)}
$$

provided the limit in probability exists. In this case we will say that the improper stochastic integral is definable, see Sato [20]. Consider a mapping between infinitely divisible distributions $\mu \mapsto \Xi_{\alpha}(\mu)$ given by

$$
\Xi_{\alpha}(\mu)=\mathcal{L}\left(\int_{0}^{\infty} t^{-1 / \alpha} d X_{t}^{(\mu)}\right) .
$$

Let $\mathfrak{D}\left(\Xi_{\alpha}\right)$ denote the domain of this mapping. The following characterization of $\mathfrak{D}\left(\Xi_{\alpha}\right)$ follows from Proposition 5.3 and Example 4.5 of Sato [20].

Theorem 3.1. (i) If $0<\alpha<1$, then

$$
\mathfrak{D}\left(\Xi_{\alpha}\right)=\left\{\mu=\mu_{(0, \nu, 0)_{0}} \in I\left(\mathbb{R}^{d}\right): \int_{\mathbb{R}^{d}}|x|^{\alpha} \nu(d x)<\infty\right\} .
$$

(ii) If $\alpha=1$, then

$$
\begin{aligned}
\mathfrak{D}\left(\Xi_{1}\right)= & \left\{\mu=\mu_{(0, \nu, 0)_{0}}=\mu_{(0, \nu, 0)_{1}} \in I\left(\mathbb{R}^{d}\right): \int_{\mathbb{R}^{d}}|x| \nu(d x)<\infty, \int_{\mathbb{R}^{d}} x \nu(d x)=0,\right. \\
& \left.\lim _{\varepsilon \downarrow 0} \int_{|x| \leq 1} x \log (|x| \vee \varepsilon) \nu(d x) \text { and } \lim _{T \rightarrow \infty} \int_{|x|>1} x \log (|x| \wedge T) \nu(d x) \text { exist }\right\} .
\end{aligned}
$$

(iii) If $1<\alpha<2$, then

$$
\mathfrak{D}\left(\Xi_{\alpha}\right)=\left\{\mu=\mu_{(0, \nu, 0)_{1}} \in I\left(\mathbb{R}^{d}\right): \int_{\mathbb{R}^{d}}|x|^{\alpha} \nu(d x)<\infty\right\} .
$$

Remark 3.2. Condition specifying when $\mu \in \mathfrak{D}\left(\Xi_{1}\right)$ looks complicated, but there is a simple sufficient condition. Namely, if $\int_{\mathbb{R}^{d}}|x||\log | x|| \nu(d x)<\infty, \int_{\mathbb{R}^{d}} x \nu(d x)=0$, and $\gamma=\int_{|x| \leq 1} x \nu(d x)$, then $\mu=\mu_{(0, \nu, \gamma)} \in \mathfrak{D}\left(\Xi_{1}\right)$.

The next three theorems are main results of this paper. The first one shows that every strictly stable law can be represented as the law of a stochastic integral in (3.1), except for the case $\alpha=1$, when the representation is up to a well specified shift 
parameter. The second one connects the integral and series representations of stable distributions. The third one gives a subdomain of $\mathfrak{D}\left(\Xi_{\alpha}\right)$ on which $\Xi_{\alpha}$ is one-to-one.

Theorem 3.3. Let $0<\alpha<2$.

(i) When $\alpha \neq 1$, we have

$$
\Xi_{\alpha}\left(\mathfrak{D}\left(\Xi_{\alpha}\right)\right)=S_{\alpha}^{0}\left(\mathbb{R}^{d}\right)
$$

(ii) When $\alpha=1$, we have

$$
\Xi_{1}\left(\mathfrak{D}\left(\Xi_{1}\right)\right)=\left\{\mu \in S_{1}^{0}\left(\mathbb{R}^{d}\right): \tau \in \operatorname{span} \operatorname{supp}\left(\lambda_{1}\right)\right\},
$$

where, respectively, $\lambda_{1}$ and $\tau$ are the spectral measure and the shift of $\mu$ given by (2.3). Here $\operatorname{supp}\left(\lambda_{1}\right)$ denotes the support of $\lambda_{1}$. If $\lambda_{1}=0$, then we put $\operatorname{span} \operatorname{supp}\left(\lambda_{1}\right)=\{0\}$ by convention.

Let $\mathrm{CP}_{0}\left(\mathbb{R}^{d}\right)$ denote the class of compound Poisson distributions on $\mathbb{R}^{d} ; \mathrm{CP}_{0}\left(\mathbb{R}^{d}\right)=$ $\left\{\mu_{(0, \nu, 0)_{0}}: \nu\left(\mathbb{R}^{d}\right)<\infty\right\}$.

Theorem 3.4. Let $\mu=\mu_{(0, \nu, 0)_{0}}$ be a compound Poisson distribution on $\mathbb{R}^{d}$ and let $\left\{X_{t}^{(\mu)}\right\}$ be the associated Lévy process. Hence $X_{t}^{(\mu)}=\sum_{j: \tau_{j} \leq t} V_{j}$, where $\left\{\tau_{j}\right\}$ is a sequence of arrival times in a Poisson process with rate $\theta=\nu\left(\mathbb{R}^{d}\right)$ and $\left\{V_{j}\right\}$ is an i.i.d. sequence in $\mathbb{R}^{d}$ with the common distribution $\theta^{-1} \nu$, independent of $\left\{\tau_{j}\right\}$. Then

$$
\int_{0}^{\infty} t^{-1 / \alpha} d X_{t}^{(\mu)}=\sum_{j=1}^{\infty} \tau_{j}^{-1 / \alpha} V_{j}
$$

in the sense that the integral is definable if and only if the series converges a.s. and then the equality holds a.s. Consequently, the series in (3.3) converges a.s. if and only if $\mu_{\left(0, \mathcal{L}\left(V_{1}\right), 0\right)_{0}} \in \mathfrak{D}\left(\Xi_{\alpha}\right)$. Furthermore, $\Xi_{\alpha}\left(\mathfrak{D}\left(\Xi_{\alpha}\right) \cap \mathrm{CP}_{0}\left(\mathbb{R}^{d}\right)\right)$ is a subclass of $S_{\alpha}^{0}\left(\mathbb{R}^{d}\right)$ consisting of distributions representable by either side of 3.3 . We have, when $0<\alpha<1$,

$$
\Xi_{\alpha}\left(\mathfrak{D}\left(\Xi_{\alpha}\right) \cap \mathrm{CP}_{0}\left(\mathbb{R}^{d}\right)\right)=S_{\alpha}^{0}\left(\mathbb{R}^{d}\right)
$$

when $\alpha=1$,

$$
\Xi_{1}\left(\mathfrak{D}\left(\Xi_{1}\right) \cap \mathrm{CP}_{0}\left(\mathbb{R}^{d}\right)\right)=\left\{\mu \in S_{1}^{0}\left(\mathbb{R}^{d}\right): \tau \in \operatorname{span} \operatorname{supp}\left(\lambda_{1}\right)\right\} ;
$$

and when $1<\alpha<2$,

$$
\begin{aligned}
(3.6) & \Xi_{\alpha}\left(\mathfrak{D}\left(\Xi_{\alpha}\right) \cap \mathrm{CP}_{0}\left(\mathbb{R}^{d}\right)\right) \\
& =\left\{\mu \in S_{\alpha}^{0}\left(\mathbb{R}^{d}\right): \exists q(\xi)>0, \int_{S} q(\xi) \xi \lambda_{1}(d \xi)=0 \text { and } \int_{S} q(\xi)^{\frac{\alpha}{\alpha-1}} \lambda_{1}(d \xi)<\infty\right\}
\end{aligned}
$$




$$
\varsubsetneqq S_{\alpha}^{0}\left(\mathbb{R}^{d}\right) .
$$

In the above, respectively, $\lambda_{1}$ and $\tau$ are the spectral measure and the shift of $\mu$ given by 2.2 -2.3 .

The following example sheds some light on the nature of (3.6).

Example 3.5. Let $\mu \in S_{\alpha}^{0}\left(\mathbb{R}^{2}\right), 1<\alpha<2$, have the spectral measure $\lambda_{1}$ supported by three vertices $\xi_{1}, \xi_{2}, \xi_{3} \in S$ of a proper triangle $\Delta$. Then

$$
\begin{aligned}
\mu \text { is representable by either side of } 3.3) & \Leftrightarrow \mu \in \Xi_{\alpha}\left(\mathfrak{D}\left(\Xi_{\alpha}\right) \cap \mathrm{CP}_{0}\left(\mathbb{R}^{2}\right)\right) \\
& \Leftrightarrow \Delta \text { is an acute triangle. }
\end{aligned}
$$

Indeed, $\Delta$ is an acute triangle if and only if 0 belongs to the interior of $\Delta$, that is, $p_{1} \xi_{1}+p_{2} \xi_{2}+p_{3} \xi_{3}=0$ for some $p_{1}, p_{2}, p_{3}>0$. Define a function $q$ on $S$ by $q\left(\xi_{i}\right)=p_{i} / \lambda_{1}\left(\left\{\xi_{i}\right\}\right), i=1,2,3$, and let $q(\xi)=1$ otherwise. Such function satisfies (3.6). The converse is clear.

Let $\mathrm{CP}_{0}(S)$ stand for the totality of compound Poisson distributions on $\mathbb{R}^{d}$ with finite Lévy measure supported on $S$ and let $\mathrm{CP}_{1}(S)$ be the totality of infinitely divisible distributions on $\mathbb{R}^{d}$ with Gaussian covariance matrix 0 , finite Lévy measure supported on $S$ and mean 0 . Then $\mathrm{CP}_{0}(S) \subset \mathfrak{D}\left(\Xi_{\alpha}\right)$ for $0<\alpha<1, \mathrm{CP}_{0}(S) \cap \mathrm{CP}_{1}(S) \subset \mathfrak{D}\left(\Xi_{1}\right)$, and $\mathrm{CP}_{1}(S) \subset \mathfrak{D}\left(\Xi_{\alpha}\right)$ for $1<\alpha<2$.

Remark 3.6. The mappings $\Xi_{\alpha}, 0<\alpha<2$, are not injective. Let us prove it in the case $\alpha=1$; the proof for $\alpha \neq 1$ is similar. Let $\widetilde{\mu}=\widetilde{\mu}_{(0, \widetilde{\nu}, 0)} \in \Xi_{1}\left(\mathfrak{D}\left(\Xi_{1}\right)\right)$. Then

$$
\begin{aligned}
\widetilde{\nu}(B) & =\int_{S} \widetilde{\lambda}(d \xi) \int_{0}^{\infty} \mathbb{1}_{B}(r \xi) r^{-2} d r \\
& =\int_{0}^{\infty} d u \int_{S} \tilde{\lambda}(d \xi) \int_{0}^{\infty} \mathbb{1}_{B}\left(u^{-1} r \xi\right) \delta_{1}(d r) \\
& =\int_{0}^{\infty} d u \int_{S} \tilde{\lambda}(d \xi) \int_{0}^{\infty} \mathbb{1}_{B}\left(u^{-1} r \xi\right) 2^{-1} \delta_{2}(d r) .
\end{aligned}
$$

Let $\mu_{1}$ and $\mu_{2}$ have the Lévy-Khintchine triplets $\left(0, \nu_{1}, 0\right)$ and $\left(0, \nu_{2}, 0\right)$, where $\nu_{1}$ and $\nu_{2}$ have polar decompositions $\left(\widetilde{\lambda}, \delta_{1}\right)$ and $\left(\widetilde{\lambda}, 2^{-1} \delta_{2}\right)$, respectively. Then $\mu_{1}, \mu_{2} \in$ $\mathfrak{D}\left(\Xi_{1}\right), \mu_{1} \neq \mu_{2}$ and $\Xi_{1}\left(\mu_{1}\right)=\Xi_{1}\left(\mu_{2}\right)=\widetilde{\mu}$. See also Remark 6.4 of Barndorff-Nielsen et al. [6]. However, as shown in what follows, the restrictions $\left.\Xi_{\alpha}\right|_{\mathrm{CP}_{0}(S)}$ with $0<\alpha<1$, $\left.\Xi_{1}\right|_{\mathrm{CP}_{0}(S) \cap \mathrm{CP}_{1}(S)}$ and $\left.\Xi_{\alpha}\right|_{\mathrm{CP}_{1}(S)}$ with $1<\alpha<2$ are injective. 
Theorem 3.7. (i) When $0<\alpha<1$, we have

$$
\Xi_{\alpha}\left(\mathrm{CP}_{0}(S)\right)=S_{\alpha}^{0}\left(\mathbb{R}^{d}\right)
$$

and the restriction $\left.\Xi_{\alpha}\right|_{\mathrm{CP}_{0}(S)}$ is injective. Especially, in the case $d=1$,

$$
S_{\alpha}^{0}(\mathbb{R})=\left\{\mathcal{L}\left(\int_{0}^{\infty} t^{-1 / \alpha} d\left(N_{1}(a t)-N_{2}(b t)\right)\right): a, b \geq 0\right\},
$$

where $\left\{N_{1}(t)\right\}$ and $\left\{N_{2}(t)\right\}$ are independent Poisson processes with unit rate.

(ii) When $\alpha=1$, we have

$$
\left.\Xi_{1}\left(\mathrm{CP}_{0}(S) \cap \mathrm{CP}_{1}(S)\right)=\left\{\mu \in S_{1}^{0}\left(\mathbb{R}^{d}\right) \text { : the shift parameter } \tau \text { in } 2.3\right) \text { is } 0\right\}
$$

and the restriction $\left.\Xi_{1}\right|_{\mathrm{CP}_{0}(S) \cap C \mathrm{P}_{1}(S)}$ is injective. Especially, in the case $d=1$,

$$
\left\{\mu \in S_{1}^{0}(\mathbb{R}): \tau=0\right\}=\left\{\mathcal{L}\left(\int_{0}^{\infty} t^{-1} d\left(N_{1}(a t)-N_{2}(a t)\right)\right): a \geq 0\right\} .
$$

(iii) When $1<\alpha<2$, we have

$$
\Xi_{\alpha}\left(\mathrm{CP}_{1}(S)\right)=S_{\alpha}^{0}\left(\mathbb{R}^{d}\right)
$$

and the restriction $\left.\Xi_{\alpha}\right|_{\mathrm{CP}_{1}(S)}$ the injective. Especially, in the case $d=1$,

$$
S_{\alpha}^{0}(\mathbb{R})=\left\{\mathcal{L}\left(\int_{0}^{\infty} t^{-1 / \alpha} d\left(N_{1}(a t)-N_{2}(b t)-(a-b) t\right)\right): a, b \geq 0\right\} .
$$

In the case (ii) of Theorem 3.7, the range of $\left.\Xi_{1}\right|_{\mathrm{CP}_{0}(S) \cap \mathrm{CP}_{1}(S)}$ is smaller than that of $\Xi_{1}$. Hence there is a subdomain $\mathfrak{D}$ such that $\operatorname{CP}_{0}(S) \cap \mathrm{CP}_{1}(S) \varsubsetneqq \mathfrak{D} \varsubsetneqq \mathfrak{D}\left(\Xi_{1}\right)$, $\Xi_{1}(\mathfrak{D})=\Xi_{1}\left(\mathfrak{D}\left(\Xi_{1}\right)\right)$ and the restriction $\left.\Xi_{1}\right|_{\mathfrak{D}}$ is injective. It is an interesting question to characterize the class $\mathfrak{D}$.

\section{Proofs}

Proof of Theorem 3.1. Statements (i) and (iii) follow from Proposition 5.3 and Example 4.5 of Sato [20], as does (ii), because

$$
\int_{\varepsilon}^{1} t^{-1} d t \int_{|x|<t} x \nu(d x)=\int_{|x| \leq 1} x \log (|x| \vee \varepsilon) \nu(d x) .
$$

and

$$
\int_{1}^{T} t^{-1} d t \int_{|x|>t} x \nu(d x)=\int_{|x|>1} x \log (|x| \wedge T) \nu(d x)
$$

The condition $\int|x| \nu(d x)<\infty$ justifies the interchange of the order of integration in these integrals.

The following lemma is needed for the proof of the next theorem. 
Lemma 4.1. Let $\lambda$ be a non-zero finite measure on $S$. Then, for any a $\in \mathbb{R}^{d}$, $a \in \operatorname{span} \operatorname{supp}(\lambda)$ if and only if $a=\int_{S} \xi f(\xi) \lambda(d \xi)$ for some $f \in L^{\infty}(S, \lambda)$.

Proof. Consider a linear subspace $H$ of $\mathbb{R}^{d}$ given by

$$
H=\left\{\int_{S} \xi f(\xi) \lambda(d \xi): f \in L^{\infty}(S, \lambda)\right\}
$$

and let $H^{\perp}$ be its orthogonal complement. Let $K=\operatorname{span} \operatorname{supp}(\lambda)$ and $K^{\perp}$ be the orthogonal complement of $K$. If $p \in H^{\perp}$, then

$$
\int_{S}\langle p, \xi\rangle f(\xi) \lambda(d \xi)=\left\langle p, \int_{S} \xi f(\xi) \lambda(d \xi)\right\rangle=0
$$

for all $f \in L^{\infty}(S, \lambda)$, which implies $\langle p, \xi\rangle=0$ for $\lambda$-almost all $\xi$. Hence $p \in K^{\perp}$. The converse inclusion, $K^{\perp} \subset H^{\perp}$ is obvious from the displayed equality, so that $H^{\perp}=K^{\perp}$. This proves $H=K$.

Proof of Theorem 3.3. We first show that $\Xi_{\alpha}\left(\mathfrak{D}\left(\Xi_{\alpha}\right)\right) \subset S_{\alpha}^{0}\left(\mathbb{R}^{d}\right)$ for all $\alpha \in(0,2)$. It is enough to prove the strict stability of $Y:=\int_{0}^{\infty} t^{-1 / \alpha} d X_{t}^{(\mu)}$ when $\mu \in \mathfrak{D}\left(\Xi_{\alpha}\right)$. Let $\bar{Y}$ be an independent copy of $Y$. Then $\bar{Y} \stackrel{\mathrm{d}}{=} \int_{0}^{\infty} t^{-1 / \alpha} d \bar{X}_{t}^{(\mu)}$ with an independent copy $\left\{\bar{X}_{t}^{(\mu)}\right\}$ of $\left\{X_{t}^{(\mu)}\right\}$. For any $c_{1}, c_{2}>0$, we have

$$
\begin{aligned}
c_{1} Y & +c_{2} \bar{Y} \\
& =\int_{0}^{\infty}\left(c_{1}^{-\alpha} t\right)^{-1 / \alpha} d X_{t}^{(\mu)}+\int_{0}^{\infty}\left(c_{2}^{-\alpha} t\right)^{-1 / \alpha} d \bar{X}_{t}^{(\mu)} \\
& =\int_{0}^{\infty} s^{-1 / \alpha} d X_{c_{1}^{\alpha} s}^{(\mu)}+\int_{0}^{\infty} s^{-1 / \alpha} d \bar{X}_{c_{2}^{\alpha} s}^{(\mu)} \stackrel{\mathrm{d}}{=} \int_{0}^{\infty} s^{-1 / \alpha} d\left(X_{s}^{\left(\mu_{1}^{c_{1}^{\alpha}}\right)}+\bar{X}_{s}^{\left(\mu^{c_{2}^{\alpha}}\right)}\right) \\
& \stackrel{\mathrm{d}}{=} \int_{0}^{\infty} s^{-1 / \alpha} d X_{s}^{\left(\mu_{1}^{c_{1}^{\alpha}} * \mu^{c_{2}^{\alpha}}\right)} \stackrel{\mathrm{d}}{=} \int_{0}^{\infty} s^{-1 / \alpha} d X_{s}^{\left(\mu^{\left.c_{1}^{\alpha}+c_{2}^{\alpha}\right)}\right.} \\
& \stackrel{\mathrm{d}}{=} \int_{0}^{\infty} s^{-1 / \alpha} d X_{\left(c_{1}^{\alpha}+c_{2}^{\alpha}\right) s}^{(\mu)}=\int_{0}^{\infty}\left(\left(c_{1}^{\alpha}+c_{2}^{\alpha}\right)^{-1} t\right)^{-1 / \alpha} d X_{t}^{(\mu)} \\
& =\left(c_{1}^{\alpha}+c_{2}^{\alpha}\right)^{1 / \alpha} \int_{0}^{\infty} t^{-1 / \alpha} d X_{t}^{(\mu)}=\left(c_{1}^{\alpha}+c_{2}^{\alpha}\right)^{1 / \alpha} Y .
\end{aligned}
$$

This shows the strict stability of $Y$.

We will also need the following relations. If $\mu=\mu_{(0, \nu, \gamma)} \in \mathfrak{D}\left(\Xi_{\alpha}\right)$, then $\Xi_{\alpha}(\mu)=$ $\widetilde{\mu}=\widetilde{\mu}_{(0, \widetilde{\nu}, \widetilde{\gamma})}$ where

$$
\begin{aligned}
\widetilde{\nu}(B) & =\int_{0}^{\infty} \int_{\mathbb{R}^{d}} \mathbb{1}_{B}\left(t^{-1 / \alpha} x\right) \nu(d x) d t \\
& =\int_{\mathbb{R}^{d}} \int_{0}^{\infty} \mathbb{1}_{B}\left(r \frac{x}{|x|}\right) \alpha r^{-\alpha-1}|x|^{\alpha} d r \nu(d x)
\end{aligned}
$$




$$
=\int_{S} \lambda(d \xi) \int_{0}^{\infty} \mathbb{1}_{B}(r \xi) r^{-\alpha-1} d r
$$

with

$$
\lambda(B)=\alpha \int_{\mathbb{R}^{d}} \mathbb{1}_{B}\left(\frac{x}{|x|}\right)|x|^{\alpha} \nu(d x),
$$

and

$$
\widetilde{\gamma}=\lim _{\varepsilon \downarrow 0, T \uparrow \infty} \int_{\varepsilon}^{T} t^{-1 / \alpha} d t\left(\gamma+\int_{\mathbb{R}^{d}} x\left(\mathbb{1}_{\left\{\left|t^{-1 / \alpha} x\right| \leq 1\right\}}-\mathbb{1}_{\{|x| \leq 1\}}\right) \nu(d x)\right)
$$

It follows that the spectral measure $\lambda_{1}$ of $\widetilde{\mu}$ is given by $\lambda_{1}=\left|\Gamma(-\alpha) \cos \frac{\pi \alpha}{2}\right| \cdot \lambda$ when $\alpha \neq 1$ and $\lambda_{1}=\frac{\pi}{2} \lambda$ when $\alpha=1$, where $\lambda$ is given by (4.2); see the proof of Theorem 14.10 in Sato [18].

(i) $(\alpha \neq 1)$. We only need to show that $S_{\alpha}^{0}\left(\mathbb{R}^{d}\right) \subset \Xi_{\alpha}\left(\mathfrak{D}\left(\Xi_{\alpha}\right)\right)$.

Case $0<\alpha<1$ : If $\widetilde{\mu}=\widetilde{\mu}_{\left(0, \widetilde{\nu}, \widetilde{\gamma}^{0}\right)_{0}} \in S_{\alpha}^{0}\left(\mathbb{R}^{d}\right)$ then $\widetilde{\gamma}^{0}=0$. Take $\mu=\mu_{(0, \nu, 0)_{0}}$ with $\nu=\alpha^{-1}\left|\Gamma(-\alpha) \cos \frac{\pi \alpha}{2}\right|^{-1} \lambda_{1}$, where $\lambda_{1}$ is the spectral measure of $\widetilde{\mu}$. Then $\mu \in \mathfrak{D}\left(\Xi_{\alpha}\right)$ and, using 4.1 -4.3), it is easy to check that $\Xi_{\alpha}(\mu)=\widetilde{\mu}$.

Case $1<\alpha<2$ : If $\widetilde{\mu}=\widetilde{\mu}_{\left(0, \widetilde{\nu}, \widetilde{\gamma}^{1}\right)_{1}} \in S_{\alpha}^{0}\left(\mathbb{R}^{d}\right)$ then $\widetilde{\gamma}^{1}=0$. Take $\mu=\mu_{(0, \nu, 0)_{1}}$ with $\nu=\alpha^{-1}\left|\Gamma(-\alpha) \cos \frac{\pi \alpha}{2}\right|^{-1} \lambda_{1}$, where $\lambda_{1}$ is the spectral measure of $\widetilde{\mu}$. Then $\mu \in \mathfrak{D}\left(\Xi_{\alpha}\right)$ and, similarly as above, we verify that $\Xi_{\alpha}(\mu)=\widetilde{\mu}$.

(ii) $(\alpha=1)$. This case is more delicate and its proof is more involved. Let $\mu_{1} \in S_{1}^{0}\left(\mathbb{R}^{d}\right)$ be given by 2.3$)$ with $\int_{S} \xi \lambda_{1}(d \xi)=0$ and $\tau \in \operatorname{span} \operatorname{supp}\left(\lambda_{1}\right)$. Put $\lambda=\frac{2}{\pi} \lambda_{1}$.

By Lemma 4.1 there is an $f \in L^{\infty}(S, \lambda)$ such that $\tau=\int_{S} \xi f(\xi) \lambda(d \xi)$. Put $g(\xi)=e^{-f(\xi)}$. Then $\varepsilon_{0}<g(\xi)<T_{0} \lambda$-a.e. $\xi \in S$ for some $0<\varepsilon_{0}<1<T_{0}$ and

$$
\tau=-\int_{S} \xi \log g(\xi) \lambda(d \xi)
$$

Define a measure $\nu$ on $\mathbb{R}^{d}$ by

$$
\nu(B)=\int_{S} \lambda(d \xi) \int_{0}^{\infty} \mathbb{1}_{B}(r \xi) \frac{1}{g(\xi)} \delta_{g(\xi)}(d r)=\int_{S} \mathbb{1}_{B}(g(\xi) \xi) \frac{1}{g(\xi)} \lambda(d \xi) .
$$

Notice that $\nu$ is a finite measure concentrated on the annulus $\left\{\varepsilon_{0}<|x|<T_{0}\right\}$ Therefore, it clearly satisfies the first, third and forth conditions on $\nu$ of Theorem 3.1(ii). The second condition is also immediate as

$$
\int_{\mathbb{R}^{d}} x \nu(d x)=\int_{S} \xi \lambda(d \xi)=0
$$


Thus $\mu=\mu_{(0, \nu, 0)_{0}}=\mu_{(0, \nu, \gamma)} \in \mathfrak{D}\left(\Xi_{1}\right)$, where $\gamma=\int_{|x| \leq 1} x \nu(d x)$. Consider $\Xi_{1}(\mu)=$ $\widetilde{\mu}_{(0, \widetilde{\nu}, \widetilde{\gamma})}$. We have

$$
\begin{aligned}
\widetilde{\nu}(B) & =\int_{0}^{\infty} \int_{\mathbb{R}^{d}} \mathbb{1}_{B}\left(t^{-1} x\right) \nu(d x) d t \\
& =\int_{0}^{\infty} \int_{S} \mathbb{1}_{B}\left(t^{-1} g(\xi) \xi\right) \frac{1}{g(\xi)} \lambda(d \xi) d t \\
& =\int_{S} \lambda(d \xi) \int_{0}^{\infty} \mathbb{1}_{B}(r \xi) r^{-2} d r
\end{aligned}
$$

and

$$
\begin{aligned}
\widetilde{\gamma} & =\lim _{\varepsilon \downarrow 0, T \uparrow \infty} \int_{\varepsilon}^{T} t^{-1} d t\left(\gamma+\int_{\mathbb{R}^{d}} x\left(\mathbb{1}_{\left\{\left|t^{-1} x\right| \leq 1\right\}}-\mathbb{1}_{\{|x| \leq 1\}}\right) \nu(d x)\right) \\
& =\lim _{\varepsilon \downarrow 0, T \uparrow \infty} \int_{\varepsilon}^{T} t^{-1} d t \int_{\mathbb{R}^{d}} x \mathbb{1}_{\left\{\left|t^{-1} x\right| \leq 1\right\}} \nu(d x) \\
& =\lim _{\varepsilon \downarrow 0, T \uparrow \infty} \int_{\mathbb{R}^{d}} x \nu(d x) \int_{\varepsilon \vee|x|}^{T} t^{-1} d t=-\int_{S} \xi \log g(\xi) \lambda(d \xi)=\tau .
\end{aligned}
$$

Note that the shift parameter in 2.3) of $\Xi_{1}(\mu)$ is $\widetilde{\gamma}+c \int_{S} \xi \lambda(d \xi)$, where $c$ is a constant; see the proof of Theorem 14.10 in Sato [18]. Since $\int_{S} \xi \lambda(d \xi)=0$, the shift parameter of $\Xi_{1}(\mu)$ is $\widetilde{\gamma}$. Thus $\Xi_{1}(\mu) \in S_{1}\left(\mathbb{R}^{d}\right)$ has the spectral measure $\frac{\pi}{2} \lambda=\lambda_{1}$ and a shift $\tau$. This proves that $\Xi_{1}(\mu)=\mu_{1}$.

Conversely, let $\widetilde{\mu} \in \Xi_{1}\left(\mathfrak{D}\left(\Xi_{1}\right)\right)$. Then for some $\mu=\mu_{(0, \nu, \gamma)} \in \mathfrak{D}\left(\Xi_{1}\right), \widetilde{\mu}=\Xi_{\alpha}(\mu)=$ $\widetilde{\mu}_{(0, \widetilde{\nu}, \widetilde{\gamma})} \cdot \widetilde{\mu} \in S_{1}^{0}\left(\mathbb{R}^{d}\right)$ has the spectral measure $\lambda_{1}=\frac{\pi}{2} \lambda$, where

$$
\lambda(B)=\int_{\mathbb{R}^{d}} \mathbb{1}_{B}\left(\frac{x}{|x|}\right)|x| \nu(d x),
$$

and a shift

$$
\begin{aligned}
\tau & :=\lim _{\varepsilon \downarrow 0, T \uparrow \infty} \int_{\varepsilon}^{T} t^{-1} d t\left(\gamma+\int_{\mathbb{R}^{d}} x\left(\mathbb{1}_{\left\{\left|t^{-1} x\right| \leq 1\right\}}-\mathbb{1}_{\{|x| \leq 1\}}\right) \nu(d x)\right) \\
& =\lim _{\varepsilon \downarrow 0, T \uparrow \infty} \int_{\varepsilon}^{T} t^{-1} d t \int_{\mathbb{R}^{d}} x \mathbb{1}_{\left\{\left|t^{-1} x\right| \leq 1\right\}} \nu(d x) .
\end{aligned}
$$

Consider a polar decomposition 2.1) of a finite measure $\rho$, given by $\rho(d x)=|x| \nu(d x)$, into the spherical component $\lambda$, given above, and the radial component $\rho_{\xi} \cdot \rho_{\xi}$ are probability measures. We have for $0<\varepsilon<1<T$,

$$
\begin{aligned}
\tau_{\varepsilon}^{T} & :=\int_{\varepsilon}^{T} t^{-1} d t \int_{\mathbb{R}^{d}} x \mathbb{1}_{\left\{\left|t^{-1} x\right| \leq 1\right\}} \nu(d x)=\left(\int_{\varepsilon}^{1}+\int_{1}^{T}\right) t^{-1} d t \int_{\mathbb{R}^{d}} x \mathbb{1}_{\left\{\left|t^{-1} x\right| \leq 1\right\}} \nu(d x) \\
& =-\int_{|x| \leq 1} x \log (\varepsilon \vee|x|) \nu(d x)-\int_{|x|>1} x \log (T \wedge|x|) \nu(d x)
\end{aligned}
$$




$$
=-\int_{S} \xi \lambda(d \xi) \int_{0}^{\infty}\left(\log (\varepsilon \vee r) \mathbb{1}_{\{r \leq 1\}}+\log (T \wedge r) \mathbb{1}_{\{r>1\}}\right) \rho_{\xi}(d r)
$$

Since $\xi \mapsto \int_{0}^{\infty}\left(\log (\varepsilon \vee r) \mathbb{1}_{\{r \leq 1\}}+\log (T \wedge r) \mathbb{1}_{\{r>1\}}\right) \rho_{\xi}(d r)$ is a bounded function $\left(\rho_{\xi}(0, \infty)=1\right), \tau_{\varepsilon}^{T} \in \operatorname{span} \operatorname{supp}(\lambda)=\operatorname{span} \operatorname{supp}\left(\lambda_{1}\right)$ by Lemma 4.1. Thus $\tau=$ $\lim _{\varepsilon \downarrow 0, T \uparrow \infty} \tau_{\varepsilon}^{T} \in \operatorname{span} \operatorname{supp}\left(\lambda_{1}\right)$.

Finally, if $\Xi_{1}(\mu)=\delta_{\tau}$ then $\lambda_{1}=0$, so that $\nu=0$. Hence $\tau=0$ by (4.4). The proof of Theorem 3.3 is complete.

Proof of Theorem 3.4. Let $\Gamma_{j}=\theta \tau_{j}$. Then $\left\{\Gamma_{j}\right\}$ is a sequence of arrival times in a Poisson process of rate one and we can write (3.3) as

$$
\int_{0}^{\infty} t^{-1 / \alpha} d X_{t}^{(\mu)}=\theta^{1 / \alpha} \sum_{j=1}^{\infty} \Gamma_{j}^{-1 / \alpha} V_{j} .
$$

Applying Theorem 4.1 of Rosiński [16] we get that the series converges a.s. if and only if $E|V|^{\alpha}=\theta^{-1} \int_{\mathbb{R}^{d}}|x|^{\alpha} \nu(d x)<\infty$ (where $V=V_{1}$ ) and the limit

$$
a:=\lim _{T \rightarrow \infty} \int_{0}^{T} E\left[t^{-1 / \alpha} V \mathbb{1}_{\left\{\left|t^{-1 / \alpha} V\right| \leq 1\right\}}\right] d t \quad \text { exists in } \mathbb{R}^{d} .
$$

If $\alpha \neq 1$ then

$$
a=\frac{\alpha}{\alpha-1} \lim _{T \rightarrow \infty} E V \mathbb{1}_{\left\{|V| \leq T^{1 / \alpha}\right\}}\left(T^{1-1 / \alpha}-|V|^{\alpha-1}\right) .
$$

Let $0<\alpha<1$. Notice that for every $\varepsilon \in(0,1)$

$$
\begin{aligned}
\limsup _{T \rightarrow \infty} & T^{1-1 / \alpha}\left|E V \mathbb{1}_{\left\{|V| \leq T^{1 / \alpha}\right\}}\right| \leq \limsup _{T \rightarrow \infty} T^{1-1 / \alpha} E|V| \mathbb{1}_{\left\{|V| \leq \varepsilon T^{1 / \alpha}\right\}} \\
& +\limsup _{T \rightarrow \infty} T^{1-1 / \alpha} E|V| \mathbb{1}_{\left\{\varepsilon T^{1 / \alpha}<|V| \leq T^{1 / \alpha}\right\}} \\
& \leq \limsup _{T \rightarrow \infty} T^{1-1 / \alpha}\left(\varepsilon T^{1 / \alpha}\right)^{1-\alpha} E|V|^{\alpha}+\limsup _{T \rightarrow \infty} E|V|^{\alpha} 1_{\left\{\varepsilon T^{1 / \alpha}<|V| \leq T^{1 / \alpha}\right\}} \\
& =\varepsilon^{1-\alpha} E|V|^{\alpha} .
\end{aligned}
$$

Letting $\varepsilon \rightarrow 0$ we show that $\lim _{\sup _{T \rightarrow \infty}} T^{1-1 / \alpha} E V \mathbb{1}_{\left\{|V| \leq T^{1 / \alpha}\right\}}=0$. Therefore,

$$
a=\frac{\alpha}{1-\alpha} E\left[\frac{V}{|V|}|V|^{\alpha}\right] \text {. }
$$

We conclude that the series in 4.5) converges a.s. if and only if $E|V|^{\alpha}<\infty$, that is, $\mu \in \mathfrak{D}\left(\Xi_{\alpha}\right)$ when $0<\alpha<1$.

If $1<\alpha<2$ and $\mu \in \mathfrak{D}\left(\Xi_{\alpha}\right)$, then $E|V|^{\alpha}<\infty$. Since also $E V=0$ we get

$$
a=\frac{\alpha}{\alpha-1} \lim _{T \rightarrow \infty} E V \mathbb{1}_{\left\{|V| \leq T^{1 / \alpha}\right\}}\left(T^{1-1 / \alpha}-|V|^{\alpha-1}\right)
$$




$$
=\frac{\alpha}{\alpha-1} \lim _{T \rightarrow \infty} E V\left(T^{1-1 / \alpha}-|V|^{\alpha-1} \wedge T^{1-1 / \alpha}\right)=\frac{\alpha}{1-\alpha} E\left[\frac{V}{|V|}|V|^{\alpha}\right] .
$$

Thus, the series in 4.5 converges a.s. Conversely, if the series in 4.5 converges a.s. then $E|V|^{\alpha}<\infty$ and the limit in (4.7) exists. Hence

$$
\begin{aligned}
0 & =\lim _{T \rightarrow \infty}\left[E V \mathbb{1}_{\left\{|V| \leq(2 T)^{1 / \alpha}\right\}}\left((2 T)^{1-1 / \alpha}-|V|^{\alpha-1}\right)-E V \mathbb{1}_{\left\{|V| \leq T^{1 / \alpha}\right\}}\left(T^{1-1 / \alpha}-|V|^{\alpha-1}\right)\right] \\
& =\left(2^{1-1 / \alpha}-1\right) \lim _{T \rightarrow \infty} T^{1-1 / \alpha} E V \mathbb{1}_{\left\{|V| \leq T^{1 / \alpha}\right\}},
\end{aligned}
$$

which implies that $E V=0$. Thus $\mu \in \mathfrak{D}\left(\Xi_{\alpha}\right)$. We conclude that, when $\alpha \neq 1$, the series in 4.5) converges a.s. if and only if $\mu \in \mathfrak{D}\left(\Xi_{\alpha}\right)$.

Now consider $\alpha=1$. Suppose that the series in 4.5 converges a.s. Then $\int_{\mathbb{R}^{d}}|x| \nu(d x)=\theta E|V|<\infty$ and the limit 4.6

$$
a:=\lim _{T \rightarrow \infty} \int_{0}^{T} E\left[t^{-1} V \mathbb{1}_{\left\{\left|t^{-1} V\right| \leq 1\right\}}\right] d t=\lim _{T \rightarrow \infty} E V \mathbb{1}_{\{|V| \leq T\}} \log \frac{T}{|V|}
$$

exists. Therefore,

$$
\begin{aligned}
0 & =\lim _{T \rightarrow \infty}\left[E V \mathbb{1}_{\{|V| \leq 2 T\}} \log \frac{2 T}{|V|}-E V \mathbb{1}_{\{|V| \leq T\}} \log \frac{T}{|V|}\right] \\
& =\lim _{T \rightarrow \infty}\left[\log 2 E V \mathbb{1}_{\{|V| \leq 2 T\}}+E V \mathbb{1}_{\{T<|V| \leq 2 T\}} \log \frac{T}{|V|}\right]=\log 2 E V .
\end{aligned}
$$

This shows that $\int_{\mathbb{R}^{d}} x \nu(d x)=\theta E V=0$. The condition $\int_{|x| \leq 1}|x||\log | x|| \nu(x)<\infty$ obviously holds because $\nu$ is a finite measure. Then for $T>1$ we have

$$
\begin{aligned}
& \int_{|x|>1} x \log (|x| \wedge T) \nu(d x)=\int_{1<|x| \leq T} x \log |x| \nu(d x)+\log T \int_{|x|>T} x \nu(d x) \\
& =\int_{1<|x| \leq T} x \log |x| \nu(d x)-\log T \int_{|x| \leq T} x \nu(d x) \\
& =-\int_{|x| \leq T} x \log \frac{T}{|x|} \nu(d x)-\int_{|x| \leq 1} x \log |x| \nu(d x) \\
& =-\theta E V \mathbb{1}_{\{|V| \leq T\}} \log \frac{T}{|V|}-\int_{|x| \leq 1} x \log |x| \nu(d x) .
\end{aligned}
$$

Since the limit in 4.8 exists, $\lim _{T \rightarrow \infty} \int_{|x|>1} x \log (|x| \wedge T) \nu(d x)$ exists as well. Thus $\mu \in \mathfrak{D}\left(\Xi_{1}\right)$. Conversely, if $\mu \in \mathfrak{D}\left(\Xi_{1}\right)$, then $E V=0$ and the above computation shows that the limit in 4.8 exists. This completes the case $\alpha=1$.

We have proved that one side in (3.3) exists if and only if the other one does. Now we will show that they are equal. Let $N_{t}=\max \left\{j: \tau_{j} \leq t\right\}$. For any fixed 
$T>0$ we have

$$
\int_{0}^{T} t^{-1 / \alpha} d X_{t}^{(\mu)}=\sum_{j=1}^{N_{T}} \tau_{j}^{-1 / \alpha} V_{j} .
$$

The integral is definable if and only if the series converges a.s., so passing $T \rightarrow \infty$ yields the almost sure equality in 3.3 .

Relation (3.4) is immediate from the proof of Theorem 3.3(i), where we constructed for any $\mu_{1} \in S_{\alpha}^{0}\left(\mathbb{R}^{d}\right), 0<\alpha<1$, a distribution $\mu=\mu_{(0, \nu, 0)_{0}} \in \mathrm{CP}_{0}\left(\mathbb{R}^{d}\right)$ such that $\Xi_{\alpha}(\mu)=\mu_{1}$. Similarly, the proof Theorem 3.3(ii) gives for any $\mu_{1} \in S_{1}^{0}\left(\mathbb{R}^{d}\right)$ having $\tau \in \operatorname{span} \operatorname{supp}\left(\lambda_{1}\right)$, a distribution $\mu=\mu_{(0, \nu, 0)_{0}} \in \mathrm{CP}_{0}\left(\mathbb{R}^{d}\right)$ such that $\Xi_{1}(\mu)=\mu_{1}$. This shows (3.4).

Now we will prove $(3.6), 1<\alpha<2$. Let $\widetilde{\mu}=\Xi_{\alpha}(\mu)$, where $\mu \in \mathfrak{D}\left(\Xi_{\alpha}\right) \cap \mathrm{CP}_{0}\left(\mathbb{R}^{d}\right)$. Hence $\int_{\mathbb{R}^{d}}|x|^{\alpha} \nu(d x)<\infty$ and $\mu=\mu_{(0, \nu, 0)_{1}}=\mu_{(0, \nu, 0)_{0}}$, so that $\int_{\mathbb{R}^{d}} x \nu(d x)=0$ by Corollary 2.1. Moreover, $\nu\left(\mathbb{R}^{d}\right)<\infty$. Consider a polar decomposition (2.1) of $\rho(d x):=\alpha|x|^{\alpha} \nu(d x)$, where the radial components $\rho_{\xi}$ are probability measures and the spherical component $\lambda$ is given by 4.2 . Define

$$
q(\xi)=\int_{0}^{\infty} r^{1-\alpha} \rho_{\xi}(d r)
$$

Then

$$
\begin{aligned}
\int_{S} q(\xi) \xi \lambda(d \xi) & =\int_{S} \int_{0}^{\infty} \xi r^{1-\alpha} \rho_{\xi}(d r) \lambda(d \xi) \\
& =\int_{\mathbb{R}^{d}} x|x|^{-\alpha} \rho(d x)=\alpha \int_{\mathbb{R}^{d}} x \nu(d x)=0
\end{aligned}
$$

and by Jensen's inequality,

$$
\begin{aligned}
\int_{S} q(\xi)^{\frac{\alpha}{\alpha-1}} \lambda(d \xi) & =\int_{S}\left(\int_{0}^{\infty} r^{1-\alpha} \rho_{\xi}(d r)\right)^{\frac{\alpha}{\alpha-1}} \lambda(d \xi) \\
& \leq \int_{S} \int_{0}^{\infty} r^{-\alpha} \rho_{\xi}(d r) \lambda(d \xi)=\alpha \int_{\mathbb{R}^{d}} \nu(d x)<\infty .
\end{aligned}
$$

As noted in the comment following (4.2), the spectral measure $\lambda_{1}$ of $\tilde{\mu}$ is proportional to $\lambda$. Therefore, $q$ satisfies the conditions of $(3.6)$.

To prove the converse inclusion, let $\mu_{1}$ be a strictly $\alpha$-stable distribution with the spectral measure $\lambda_{1}$ such that for some function $q$ the conditions of $(3.6)$ hold. Define a measure $\nu$ by

$$
\nu(B)=c \int_{S} \lambda_{1}(d \xi) \int_{0}^{\infty} \mathbb{1}_{B}(r \xi) q(\xi)^{\frac{\alpha}{\alpha-1}} \delta_{q(\xi)^{\frac{1}{1-\alpha}}}(d r)
$$


where $c$ is a constant to be determined later. Then $\nu\left(\mathbb{R}^{d}\right)=c \int_{S} q(\xi)^{\frac{\alpha}{\alpha-1}} \lambda_{1}(d \xi)<\infty$ and

$$
\int_{\mathbb{R}^{d}}|x|^{\alpha} \nu(d x)=c \int_{S} \lambda_{1}(d \xi)<\infty
$$

Moreover,

$$
\int_{\mathbb{R}^{d}} x \nu(d x)=c \int_{S} q(\xi) \xi \lambda_{1}(d \xi)=0
$$

Thus $\mu=\mu_{(0, \nu, 0)_{1}} \in \mathfrak{D}\left(\Xi_{\alpha}\right) \cap \mathrm{CP}_{0}\left(\mathbb{R}^{d}\right)$. Finally, for any $B \in \mathcal{B}(S)$,

$$
\int_{\mathbb{R}^{d}} \mathbb{1}_{B}\left(\frac{x}{|x|}\right)|x|^{\alpha} \nu(d x)=c \int_{S} \mathbb{1}_{B}(\xi) \lambda_{1}(d \xi)=c \lambda_{1}(B) .
$$

Hence, taking $c=\left|\alpha \Gamma(-\alpha) \cos \frac{\pi \alpha}{2}\right|^{-1}$, we get $\mu_{1}=\Xi_{\alpha}(\mu)$.

The strict inclusion in 3.6 is obvious. Indeed, let $\mu \in S_{\alpha}^{0}\left(\mathbb{R}^{d}\right)$ have the spectral measure $\lambda_{1}=\delta_{v}$, where $v \in S$. Since $\int_{S} q(\xi) \xi \lambda_{1}(d \xi)=q(v) v \neq 0$ for any positive function $q, \mu \notin \Xi_{\alpha}\left(\mathfrak{D}\left(\Xi_{\alpha}\right) \cap \mathrm{CP}_{0}\left(\mathbb{R}^{d}\right)\right)$. The proof of Theorem 3.4 is complete.

Proof of Theorem 3.7. (i) The inclusion $\Xi_{\alpha}\left(\mathrm{CP}_{0}(S)\right) \subset S_{\alpha}^{0}\left(\mathbb{R}^{d}\right)$ is obvious by Theorem 3.3 . Let $\tilde{\mu} \in S_{\alpha}^{0}\left(\mathbb{R}^{d}\right)$. In the proof of Theorem 3.3 we constructed a distribution $\mu=\mu_{(0, \nu, 0)_{0}} \in \mathrm{CP}_{0}(S)$ such that $\Xi_{\alpha}(\mu)=\widetilde{\mu}$. Thus $\Xi_{\alpha}\left(\mathrm{CP}_{0}(S)\right) \supset S_{\alpha}^{0}\left(\mathbb{R}^{d}\right)$.

Let $\mu_{1}=\mu_{1\left(0, \nu_{1}, 0\right)_{0}}, \mu_{2}=\mu_{2\left(0, \nu_{2}, 0\right)_{0}} \in \mathrm{CP}_{0}(S)$ and $\Xi_{\alpha}\left(\mu_{1}\right)=\Xi_{\alpha}\left(\mu_{2}\right)$. Then by 4.1) and (4.2), the spectral measure of $\Xi_{\alpha}\left(\mu_{1}\right)$ is $\alpha\left|\Gamma(-\alpha) \cos \frac{\pi \alpha}{2}\right| \nu_{1}$. By the uniqueness of spectral measure, we have $\alpha\left|\Gamma(-\alpha) \cos \frac{\pi \alpha}{2}\right| \nu_{1}=\alpha\left|\Gamma(-\alpha) \cos \frac{\pi \alpha}{2}\right| \nu_{2}$. Thus $\mu_{1}=\mu_{2}$.

In the case $d=1, S=\{-1,1\}$ and hence

$$
\Xi_{\alpha}\left(\mathrm{CP}_{0}(S)\right)=\left\{\mathcal{L}\left(\int_{0}^{\infty} t^{-1 / \alpha} d\left(N_{1}(a t)-N_{2}(b t)\right)\right): a, b \geq 0\right\} .
$$

(ii) Let $\widetilde{\mu}=\widetilde{\mu}_{(0, \widetilde{\nu}, \widetilde{\gamma})} \in \Xi_{1}\left(\mathrm{CP}_{0}(S) \cap \mathrm{CP}_{1}(S)\right)$. Then $\widetilde{\mu}=\Xi_{1}(\mu) \in S_{1}^{0}\left(\mathbb{R}^{d}\right)$ for some $\mu=\mu_{(0, \nu, 0)_{0}} \in \mathrm{CP}_{0}(S) \cap \mathrm{CP}_{1}(S)$. Then $\int_{S} \xi \nu(d \xi)=0$. Therefore

$$
\widetilde{\gamma}=\lim _{\varepsilon \downarrow 0, T \uparrow \infty} \int_{\varepsilon}^{T} t^{-1} d t \int_{\mathbb{R}^{d}} x \mathbb{1}_{\left\{\left|t^{-1} x\right| \leq 1\right\}} \nu(d x)=\lim _{\varepsilon \downarrow 0, T \uparrow \infty} \int_{\varepsilon}^{T} t^{-1} \mathbb{1}_{\{t \geq 1\}} d t \int_{S} \xi \nu(d \xi)=0 .
$$

Hence the shift parameter $\tau$ in 2.3 of $\widetilde{\mu}$ is 0 .

Conversely, let $\tilde{\mu} \in S_{1}^{0}\left(\mathbb{R}^{d}\right)$ have the shift parameter 0 . Take $\mu=\mu_{(0, \nu, 0)_{0}}$ with $\nu=\frac{2}{\pi} \lambda_{1}$, where $\lambda_{1}$ is the spectral measure of $\widetilde{\mu}$. Then $\mu \in \mathrm{CP}_{0}(S) \cap \mathrm{CP}_{1}(S)$ and $\Xi_{1}(\mu)=\widetilde{\mu}$.

The rest of the proof of this case is similar to the case (i).

(iii) This case is similar to the case (i). 


\section{Final Remarks}

The following proposition and remark are concerned with the limits of ranges of iterations of the mappings $\Xi_{\alpha}, 0<\alpha<2$. The composition $\Xi_{\alpha}^{2}=\Xi_{\alpha} \circ \Xi_{\alpha}$ is defined on the domain $\mathfrak{D}\left(\Xi_{\alpha}^{2}\right)=\left\{\mu \in \mathfrak{D}\left(\Xi_{\alpha}\right): \Xi_{\alpha}(\mu) \in \mathfrak{D}\left(\Xi_{\alpha}\right)\right\}$.

Proposition 5.1. Let $0<\alpha<2$. Then $\mathfrak{D}\left(\Xi_{\alpha}^{2}\right)=\left\{\delta_{0}\right\}$, so that

$$
\Xi_{\alpha}^{m}\left(\mathfrak{D}\left(\Xi_{\alpha}^{m}\right)\right)=\left\{\delta_{0}\right\} \quad \text { for every } m \geq 2
$$

Proof. Let $\mu \in \mathfrak{D}\left(\Xi_{\alpha}^{2}\right)$ have the Lévy measure $\nu$. Since $\Xi_{\alpha}(\mu) \in \mathfrak{D}\left(\Xi_{\alpha}\right)$, its Lévy measure $\widetilde{\nu}$ has finite $\alpha$-th moment by Theorem 3.1. From 4.1) this is only possible when $\nu=0$. Hence $\mu$ is a point mass distribution belonging to $\mathfrak{D}\left(\Xi_{\alpha}\right)$. By Theorem 3.1 (or simply by (3.1) $), \mu=\delta_{0}$.

Remark 5.2. Given a stochastic integral mapping $\Phi_{f}$ in 1.1), an interesting problem is to determine the limit $\bigcap_{m=1}^{\infty} \Phi_{f}^{m}\left(\mathfrak{D}\left(\Phi_{f}^{m}\right)\right)$. Recently, for many integrands $f$, the descriptions of $\bigcap_{m=1}^{\infty} \Phi_{f}^{m}\left(\mathfrak{D}\left(\Phi_{f}^{m}\right)\right)$ have been obtained. In many cases, $\bigcap_{m=1}^{\infty} \Phi_{f}^{m}\left(\mathfrak{D}\left(\Phi_{f}^{m}\right)\right)$ is the class $L_{\infty}\left(\mathbb{R}^{d}\right)$ of completely selfdecomposable distributions or its subclasses; see Maejima and Sato [10], Maejima and Ueda [12, 13], Aoyama et al. [1], Sato [22, 23]. Also, in some cases, $\bigcap_{m=1}^{\infty} \Phi_{f}^{m}\left(\mathfrak{D}\left(\Phi_{f}^{m}\right)\right)$ is the class $L_{\infty}\left(b, \mathbb{R}^{d}\right)$ of completely semiselfdecomposable distributions with span $b>1$, which is the closure of the class of semi-stable distributions with span $b$ under convolution and weak convergence; see Maejima and Ueda [11]. Other examples are found in Section 4 of Sato [22]. However, except of $\Xi_{\alpha}$, we do not know any example of a stochastic integral mapping $\Phi_{f}$ satisfying $\Phi_{f}\left(\mathfrak{D}\left(\Phi_{f}\right)\right) \neq\left\{\delta_{0}\right\}$ and $\bigcap_{m=1}^{\infty} \Phi_{f}^{m}\left(\mathfrak{D}\left(\Phi_{f}^{m}\right)\right)=\left\{\delta_{0}\right\}$.

\section{REFERENCES}

[1] T. Aoyama, A. Lindner, and M. Maejima. A new family of mappings of infinitely divisible distributions related to the Goldie-Steutel-Bondesson class. Electron. J. Probab. 15 (2010), 1119-1142.

[2] T. Aoyama and M. Maejima. Characterizations of subclasses of type $G$ distributions on $\mathbb{R}^{d}$ by stochastic integral representations. Bernoulli 13 (2007), 148-160.

[3] T. Aoyama, M. Maejima, and J. Rosiński. A subclass of type $G$ selfdecomposable distributions on $\mathbb{R}^{d}$. J. Theor. Probab. 21 (2008), 14-34. 
[4] T. Aoyama, M. Maejima, and Y. Ueda. Several forms of stochastic integral representations of gamma random variables and related topics. Probab. Math. Statist. 31 (2011), 99-118.

[5] O.E. Barndorff-Nielsen, M. Maejima, and K. Sato. Some classes of multivariate infinitely divisible distributions admitting stochastic integral representations. Bernoulli 12 (2006), 1-33.

[6] O.E. Barndorff-Nielsen, J. Rosinski, and S. Thorbjørnsen. General $\Upsilon$ transformations. ALEA, Lat. Am. J. Probab. Math. Stat. 4 (2008), 131-165.

[7] K. Ichifuji and M. Maejima and Y. Ueda. Fixed points of mappings of infinitely divisible distributions on $\mathbb{R}^{d}$. Statist. Probab. Lett. 80 (2010), 1320-1328.

[8] Z.J. Jurek. Relations between the $s$-selfdecomposable and selfdecomposable measures. Ann. Probab. 13 (1985), 592-608.

[9] Z.J. Jurek and W. Vervaat. An integral representation for selfdecomposable Banach space valued random variables. Z. Wahrscheinlichkeitstheor. Verw. Geb. 62 (1983), 247-262.

[10] M. Maejima and K. Sato. The limits of nested subclasses of several classes of infinitely divisible distributions are identical with the closure of the class of stable distributions. Probab. Theory Relat. Fields 145 (2009), 119-142.

[11] M. Maejima and Y. Ueda. Stochastic integral characterizations of semiselfdecomposable distributions and related Ornstein-Uhlenbeck type processes. Commun. Stoch. Anal. 3 (2009), 349-367.

[12] M. Maejima and Y. Ueda. Compositions of mappings of infinitely divisible distributions with applications to finding the limits of some nested subclasses. Electron. Commun. Probab. 15 (2010), 227-239.

[13] M. Maejima and Y. Ueda. Nested subclasses of the class of $\alpha$-selfdecomposable distributions. Tokyo J. Math. 34 (2011), 383-406.

[14] A. Rocha-Arteaga and K. Sato. Topics in Infinitely Divisible Distributions and Lévy Processes. Aportaciones Matemáticas, Investigación 17, Sociedad Matemática Mexicana, (2003).

[15] J. Rosinski. On series representations of infinitely divisible random vectors. Ann. Probab. 18 (1990), 405-430.

[16] J. Rosiński. Series representations of Lévy processes from the perspective of point processes. In: Barndorff-Nielsen, Ole E. (ed.) et al., Lévy processes. Theory and applications. Boston: Birkhäuser. 401-415, 2001. 
[17] G. Samorodnitsky and M.S. Taqqu. Stable Non-Gaussian Random Processes. Stochastic Modeling. New York: Chapman \& Hall. Stochastic models with infinite variance.

[18] K. Sato. Lévy Processes and Infinitely Divisible Distributions. Cambridge University Press, Cambridge, 1999.

[19] K. Sato. Two families of improper stochastic integrals with respect to Lévy processes. ALEA, Lat. Am. J. Probab. Math. Stat. 1 (2006), 47-87.

[20] K. Sato. Transformations of infinitely divisible distributions via improper stochastic integrals. ALEA, Lat. Am. J. Probab. Math. Stat. 3 (2007), 67-110.

[21] K. Sato. Fractional integrals and extensions of selfdecomposability. Lecture Notes in Mathematics 2001, Lévy matters I. 1-91, Springer, 2010.

[22] K. Sato. Description of limits of ranges of iterations of stochastic integral mappings of infinitely divisible distributions. ALEA, Lat. Am. J. Probab. Math. Stat. 8 (2011), 1-17.

[23] K. Sato. Inversions of infinitely divisible distributions and conjugates of stochastic integral mappings. To appear in J. Theoret. Probab. (2013).

[24] K. Sato and Y. Ueda. Weak drifts of infinitely divisible distributions and their applications. J. Theoret. Probab. 26 (2013), 885-898.

[25] K. Sato and M. Yamazato. Operator-selfdecomposable distributions as limit distributions of processes of Ornstein-Uhlenbeck type. Stoch. Proc. Appl. 17 (1984), 73-100.

[26] S.J. Wolfe. On a continuous analogue of the stochastic difference equation $X_{n}=$ $\rho X_{n-1}+B_{n}$. Stoch. Proc. Appl. 12 (1982), 301-312. 